\title{
Range-finding method using diffraction gratings
}

Thomas D. DeWitt

Douglas A. Lyon

Fairfield University, dlyon@fairfield.edu

Follow this and additional works at: https://digitalcommons.fairfield.edu/engineering-facultypubs Copyright 1995 Optical Society of America

\section{Peer Reviewed}

\section{Repository Citation}

DeWitt, Thomas D. and Lyon, Douglas A., "Range-finding method using diffraction gratings" (1995).

Engineering Faculty Publications. 87.

https://digitalcommons.fairfield.edu/engineering-facultypubs/87

\section{Published Citation}

DeWitt, Thomas D., and Douglas A. Lyon. "Range-finding method using diffraction gratings." Applied optics 34, no.

14 (1995): 2510-2521.

This item has been accepted for inclusion in DigitalCommons@Fairfield by an authorized administrator of DigitalCommons@Fairfield. It is brought to you by DigitalCommons@Fairfield with permission from the rightsholder(s) and is protected by copyright and/or related rights. You are free to use this item in any way that is permitted by the copyright and related rights legislation that applies to your use. For other uses, you need to obtain permission from the rights-holder(s) directly, unless additional rights are indicated by a Creative Commons license in the record and/or on the work itself. For more information, please contact digitalcommons@fairfield.edu. 


\title{
Range-finding method using diffraction gratings
}

\author{
Thomas D. DeWitt and Douglas A. Lyon
}

\begin{abstract}
A model in geometric optics, along with some preliminary experimental results for a new range-finding method that exploits near-field diffraction phenomena found with plane gratings, is presented. Among the characteristics investigated is a magnification effect applicable to three-dimensional microscopy. A variety of embodiments of the method is disclosed, including an off-axis illumination model and a method of near-field focus compensation that takes advantage of the Scheimpflug condition.
\end{abstract}

\section{Introduction}

As a point source approaches a plane grating, its higher-order spectra shift toward its central zeroorder image. This observation can be described by the use of geometric optical models generally reserved for the Fraunhofer case of diffraction, even though observations are taking place within what is generally regarded as the Fresnel regime, that is, where the wave front striking the grating is not plane but rather is measurably spherical in shape. Our model assumes the use of a lens or, in the simple case, a pinhole aperture, that is, a perspective center, in order to form diffraction images at a focal plane.

We define the pitch of a grating as the spacing between the centers of adjacent grating slits. The phenomena disclosed here are most pronounced when a grating is used whose pitch is less than, or near to, the wavelength of the illumination incident upon the grating. This appears up to a limit at gratings of a pitch that is half that of the illumination's wavelength. We demonstrate that a magnification effect can be achieved with such gratings in the near field.

Section 2 reviews the technology of near-field range finding. It includes a brief survey of near-field rangefinding methods and a literature survey.

Subsection 3.A presents a mathematical model of a diffraction range finder with, alternatively, a perspective center and a simple lens for image formation. The assumption in this section is that the sensor and

D. Lyon is with the Department of Computer Science and Engineering, University of Bridgeport, Bridgeport, Connecticut 06601. T. D. DeWitt is with the DeWitt Brothers Tool Company, Inc., Box 83, Ancramdale, New York 12503.

Received 4 August 1994; revised manuscript received 22 December 1994

0003-6935/95/142510-12\$06.00/0

(4) 1995 Optical Society of America. the target form a line orthogonal to the grating plane. Subsection 3.B extends our analysis to a more general case in which source illumination is not restricted to a line that is strictly orthogonal to the grating plane. Section 4 compares experimental results with predictions based on the models given in Section 3. In Section 5 we offer a model for the use of the Scheimpflug condition. Section 6 is a comparison with other range-finding methods in the light of the unique features of diffraction range finding.

\section{Near-Field Range-Finding Problem}

Range-finding technologies include triangulation, focus analysis, interferometry, moiré, and time-of-flight methods such as sonar, lidar, and radar. These all can be practiced with active illumination techniques comparable with the diffraction method we detail in the sections below. The literature is replete with surveys of these technologies, and we have included a bibliography. ${ }^{1}$

Triangulation methods are the most common form of range finding in use, but their inherent limitations have stunted exploitation. All triangulation methods have zones of occlusion, particularly in the near field. The near-field blind areas are in the very region where accuracy would be greatest, given that, for triangulation and stereoscopy, accuracy is inversely proportional to target distance. Occlusion liability can be lowered by a decrease in the baseline between transmitter and receiver, thereby lowering the triangulation angle, but this results in a sacrifice of resolution. Another restriction affecting triangulation methods is that, in scanning modes intended to acquire range data over an area of view, it is inconvenient to synchronize the movement of structured illumination with the view field of the receiver. Solutions to this problem have been proposed but have not enjoyed widespread use. ${ }^{2}$ As a result, when staring arrays are used to receive, resolution is sacri- 
ficed to achieve a significant field of view over a work area.

Range finding by focus analysis has the advantage over triangulation of being monocular, that is, the source of illumination and the receiver can be coaxial. This advantage would seemingly overcome the synchronization problem for area scanning. However, the lenses used for accurate range measurements by focus analysis have relatively large primary elements because they work by minimizing depth of field. Such large instruments can be awkward to scan quickly. Adjusting focus on large lenses also carries a mechanical penalty: it is time consuming. In the extreme near field, in which microscopes can be used, the small target distances reduce the need for large primary elements. However, microscopes present their own practical limitations. These include a narrow band of range detection, a limited field of view, and an extremely short work standoff between instrument and target. Focus analysis computations can be simple for point-by-point measurements, as these require only minimizing the circle of confusion by a mechanical adjustment of the objective. However, as the overall depths to be ranged are broadened, the mechanical travel of such adjustments becomes time consuming. If a mechanically passive method is used, as might be required when the source of illumination is a projected line for profilometry, focus analysis becomes computationally expensive and less reliable because of variations in target reflectivity.

Interferometric methods of ranging can be monocular (coaxial transmitter and receiver), but they operate in only a relative coordinate space of contiguous target surfaces. Any abrupt discontinuity in a target surface produces ambiguous results. In the otherwise exquisitely sensitive wavelength interference designs, as were first shown by the MichaelsonMorely experiment and which are now routinely used for measuring the surfaces of lenses, discontinuities in target surface topology cannot be easily resolved.

Moiré methods are a form of interferometry that allows for a much coarser increment of depth measurement than classical interferometry based on the wavelength of light. Nonetheless, moiré methods suffer ambiguity for target discontinuities greater than these coarser steps of measurement. Moreover, moiré methods have all the occlusion problems associated with triangulation, and the remedy of lowering the angle between transmitter and receiver carries the penalty of resolution loss just as it does in triangulation. The primary advantage of moiré is temporal. An entire surface can be acquired in a single camera exposure, albeit the postacquisition processing can be quite time consuming.

Time-of-flight methods of range finding in their native forms do not suffer from the ambiguities of a relative coordinate system. Ultrasonic ranging has evolved into an economical method for many applications, but sonar is limited to primarily liquid and solid media for imaging. In air, the angle of dispersion of the sound beacon is too broad to form an image conveniently. Ultrasonic electronics also tend to fail at short ranges because the illumination chirp interferes with echo detection. Similar near-field blindness affects most forms of radar and lidar. Moreover, with these higher-frequency media, time-offlight measurements tax the fastest electronic detectors for small increments of range measurement. There are types of lidar that overcome some of these limitations in accuracy and near-field blindness. A detailed analysis of the modulation methods used is beyond the scope of this article. However, as a general rule, these methods do not work well in the extreme near field, under $10 \mathrm{~cm}$, and they carry an ambiguity penalty similar to interferometry methods.

\section{Mathematical Model of Diffraction Range Finding}

\section{A. Basic Model}

A diffraction grating reradiates incident energy as a large number of new point-source radiators. To an observer looking at the grating, only those wave fronts that arrive with constructive interference at the point of observation are detected. The remainder are eliminated by phase cancellation.

For a point-source radiator at infinity, the intensity maxima are perceived at angles off the normal according to

$$
\sin r=\lambda(n / p)
$$

where $r$ is angle of the received maxima, $\lambda$ is the wavelength of the incident radiation, $p$ is the diffraction pitch, and $n$ is the diffraction order, an integer.

When the incident wave front striking the grating originates off the surface normal, a second term must be considered. We will call it angle $i$, the angle of the incident wave front, and

$$
\sin i+\sin r=\lambda(n / p) .
$$

Equations (1) and (2) are referred to as the grating equations and are well-known relationships. ${ }^{3}$

Consider the model shown in Fig. 1. A pointsource radiator at $O$ is viewed through grating $G$. The range is $D$, and $d$ is the standoff between the grating and a perspective center $C$.

Using the relationship in Eq. (2) as a basis, we have shown in a prior publication ${ }^{4}$ that

$$
D=\frac{\left.d \tan r \mid 1-(n \lambda / p-\sin r)^{2}\right]^{1 / 2}}{n \lambda / p-\sin r}
$$

Equation (3) is useful because it defines range as a function of $r$, the angle at which the higher-order images are received behind the grating. However,

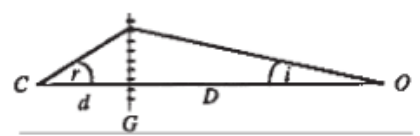

Fig. 1. Simple geometric model of diffraction. 


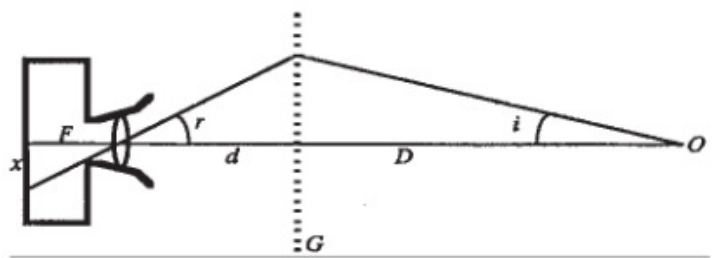

Fig. 2. Geometric model of a camera with lens and grating.

image formation requires a lens, so the equation must be further refined. Consider the model in Fig. 2. A camera lens of focal length $F$ forms an image at the focal plane. The high-order diffraction image forms at an offset, $x$, from the zero-order at the center.

Similar triangles can be identified on either side of the lens, so that

$$
\begin{aligned}
& \tan r=(x / F), \\
& \sin r=\frac{x}{\left(x^{2}+F^{2}\right)^{1 / 2}}
\end{aligned}
$$

must be true.

Substitution of Eqs. (4) and (5) into Eq. (3) yields

$$
D=(x d / F) \frac{\left\{1-\left[n \lambda / p-\frac{x}{\left(x^{2}-F^{2}\right)^{1 / 2}}\right]^{2}\right\}^{1 / 2}}{(n \lambda / p)\left(x^{2}+F^{2}\right)^{1 / 2}-x} .
$$

To our knowledge, even though Eqs. (3) and (6) fall out directly from grating equation (2), they did not appear in the literature before our publications did. ${ }^{5}$ Our claim that a target's range could be correlated to the angle subtended by its diffracted image was sufficiently novel to be awarded a basic method patent. ${ }^{6}$

\section{B. Diffraction Range Finding with Off-Axis Illumination}

Consider the configuration illustrated in Fig. 3. A laser line is projected at a relief spacing $s$ and angle $\alpha$ relative to the median line formed by $d$ and $D$. The distance from the grating plane to a target $O$ along the laser line is $D L$. Length $S$ along the grating can be determined by measured values: length $d$ and angle $r$. Line segment $D L_{0}$ can be found by similar triangle ratios. $D L$ can then be derived.

We can write

$$
D L=\frac{\kappa(d \tan r-s)}{\cos \alpha-\kappa \tan \alpha},
$$

where

$$
\kappa=\frac{\left[1-(n \lambda / p-\sin r)^{2}\right]^{1 / 2}}{n \lambda / p-\sin r} .
$$

A detailed derivation for Eqs. (7) and (8) appears in Appendix A.

An off-axis model for diffraction range finding other than the one offered here was the topic of a 1987 National Science Foundation Small Business Innova-

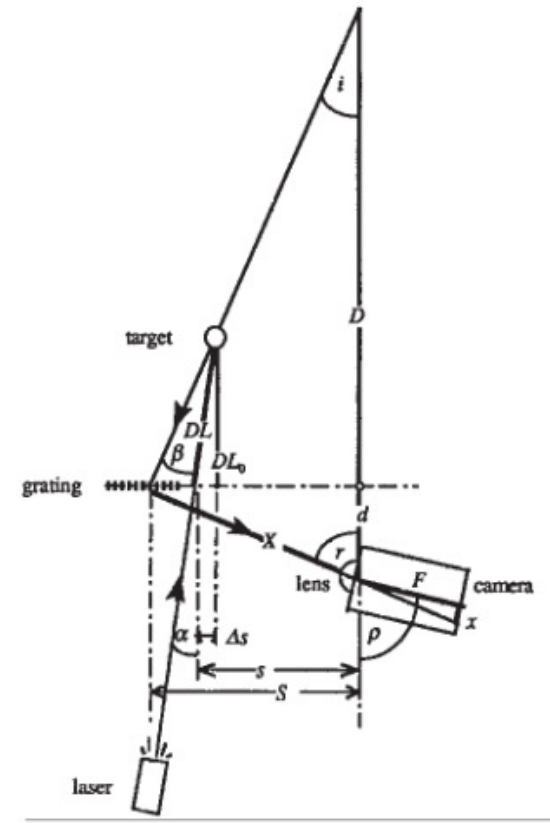

Fig. 3. General case of a diffraction range finder.

tive Research grant. ${ }^{7}$ This model did not include a perspective center for the observer, and, as a result, its mathematical derivations arrive at a set of expressions different from ours. However, this work does broach the issue of maxima intensity. With the exception of calculating the Bragg angle (for which one can anticipate high grating efficiency), we have left this topic for our future research. This is because, in part, we have a special interest in highfrequency gratings in which intensity models are particularly idiosyncratic and not categorized by closed-form relationships.

As we did for the geometric relationship (3), we can modify Eq. (7) to include a simplified camera model. A further parameter, $\rho$, is included to describe the rotation of the camera toward the diffraction image:

$$
D L=\frac{\{d \tan [\rho+\arctan (x / F)]-s\} \kappa}{\cos \alpha-\kappa \tan \alpha},
$$

where

$$
\kappa=\frac{\left(1-\left\{n \lambda / p-\left.\sin |\rho-a \tan (x / F)|\right|^{2}\right)^{1 / 2}\right.}{n \lambda / p-\sin |\rho-a \tan (x / F)|}
$$

For cases in which it is more convenient to measure $X$, the distance from the grating to the camera along its axis of view, rather than $d$, the normal from the grating plane to the lens, we can substitute

$$
d=x \cos \rho .
$$

We have coined an expression, the occlusion liability angle, for $\beta$, the difference between the illumination angle, $\alpha$, and the angle incident upon the grating, $i$ :

$$
\beta=a \sin (n \lambda / p-\sin r)-\alpha .
$$


This parameter can be used as the basis of comparison of the diffraction method with triangulation in which occlusion liability is a key performance criterion.

\section{Reconciliation of Mathematical Models with Experimentation}

We made our earliest observations by using diffraction gratings whose pitch was larger than the wavelength of the source illumination. Such gratings have practical merits when used in diffraction range finders. With nonsinusoidal groove geometries, they can produce a multiplicity of higher-order diffraction images from a single point source, allowing for redundant views that can overcome many near-field occlusion cases. The lower-order images have lower occlusion liability angles than the higher-order images do, whereas the higher-order images have greater sensitivity to range.

Consider a coarse grating with a pitch of $5555 \mathrm{~nm}$. We conducted an experiment using the bench setup illustrated in Fig. 4. A test block was used as a target. It had milled steps of $0.1 \mathrm{in} .(2.54 \mathrm{~mm})$. The target was illuminated with a 670-nm laser stripe that was projected through an open gap in the grating. (Projection through the grating itself would have produced a multiplicity of illumination stripes. This is a strategy sometimes used in triangulation devices, and a comparison with our method appears in Section 6.)

Using Eqs. (7) and (8), we can graph the received angle of diffraction versus the range, as shown in Fig. 5. The occlusion liability angles, $\beta$, are shown in the matching graph. The predicted camera image is graphed in Fig. 6. We assumed a camera at a distance $d$ of $20 \mathrm{~cm}$ to the grating, a lens with focal length $F=25 \mathrm{~mm}$, and a target range of 30 to $70 \mathrm{~mm}$. Our experimental result is shown in the matching camera recording in Fig. 6 . The image is of a test block with $0.254-\mathrm{mm}$ steps. It must be noted that the grating used to produce the image was an inexpensive embossed plastic sheet (available from Spectratek of Los Angeles).



Fig. 4. Bench test for low-frequency grating $(p=5.55 \mathrm{~mm}$, $l=670 \mathrm{~nm}$ ). Camera-to-grating distance $d=20 \mathrm{~cm}$, test block steps $2.54 \mathrm{~mm} \times 2.54 \mathrm{~mm}$
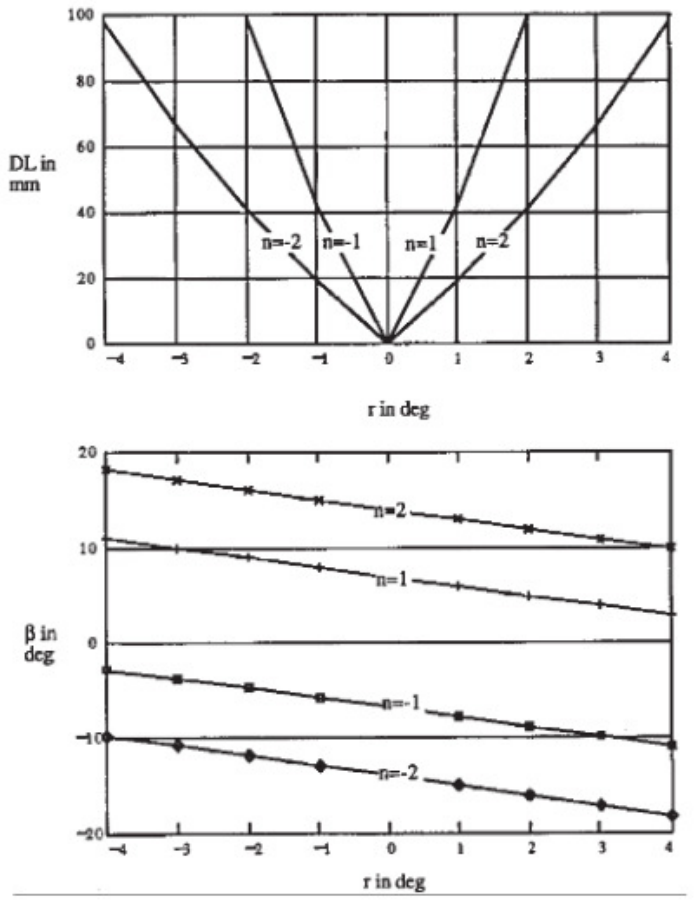

Fig. 5. Graph of range $D L$ versus angle of received diffraction image $r$ for first and second orders.

The effect of a $20^{\circ}$ off-axis rotation of the source illumination with the $5555-\mathrm{nm}$ grating is illustrated in Fig. 7. The negative orders are no longer symmetrical with the positive orders. It can be argued that this effect increases the sensitivity of a grating to range, ${ }^{8}$ but because the zero order also shifts according the principle of triangulation, the increased sensitivity is really a compound effect of both triangulation and diffraction. A camera-recorded image of our test
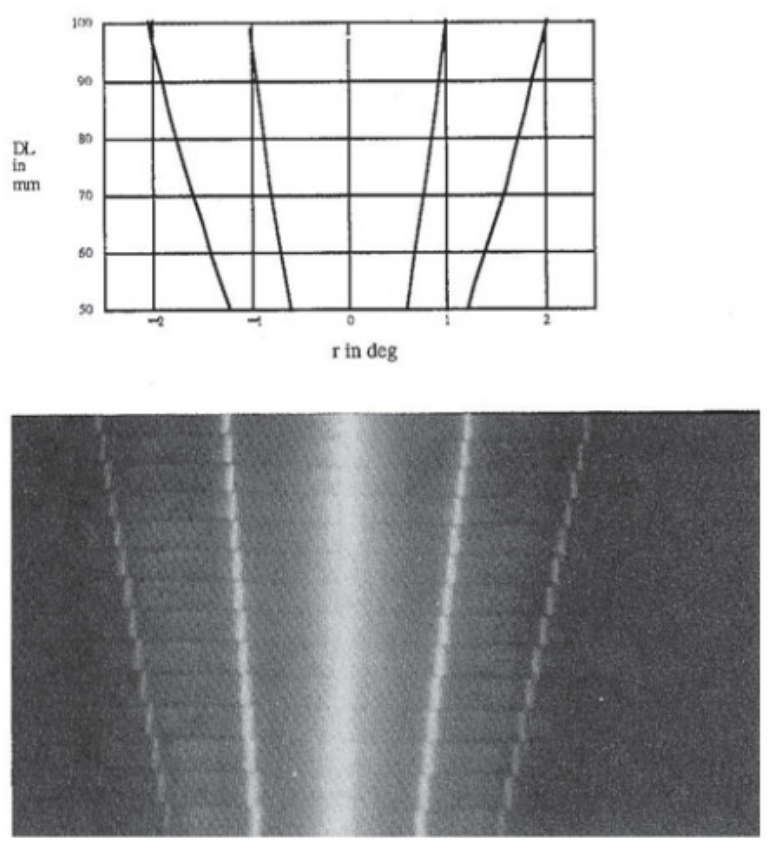

Fig. 6. Camera image of test block with low-frequency grating. 



Fig. 7. Predicted behavior of off-axis illumination and a comparison of occlusion liability angles for first and second orders.

block positioned $30 \mathrm{~mm}$ from the grating is shown in Fig. 8.

Another means of increasing grating sensitivity to range is to decrease the grating pitch relative to the wavelength of the source illumination. We illustrate the phenomenon by using a grating whose pitch is 10 times finer than that used in the two experiments above.

Consider the bench experiment shown in Fig. 9. A step test block is illuminated by a laser with a relief $s$ of $100 \mathrm{~mm}$ and a rotation of $\alpha=-22^{\circ}$. The negative rotation optimizes the sensitivity to range at the expense of occlusion liability, as graphed in Fig. 10. The sensitivity is sufficient to magnify range resolution relative to a corresponding lateral dimension. Referring to Fig. 11, we can compare predicted and actual performances for an image taken with a $25-\mathrm{mm}$ lens, where the median distance $d$ from the grating plane to the camera is $245 \mathrm{~mm}$. Each vertical line in the camera image corresponds to a length of $2.54 \mathrm{~mm}$.

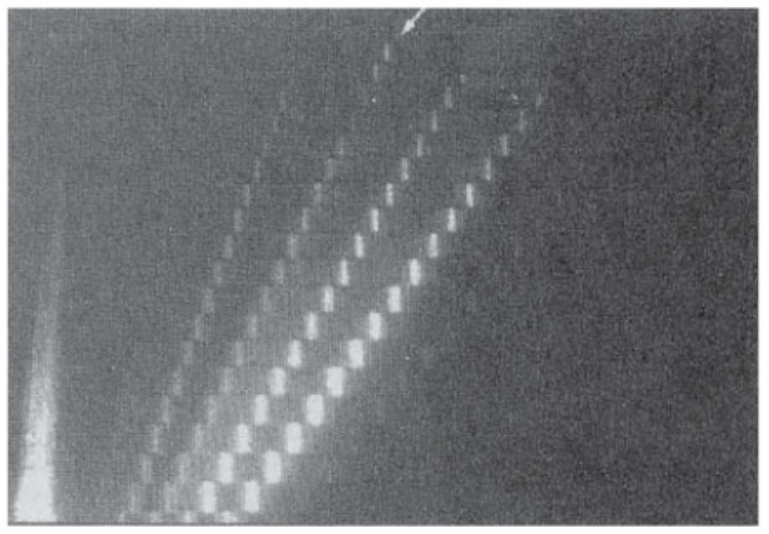

Fig. 8. Off-axis illumination with a 5555-nm grating: $\alpha=20^{\circ}$, the zero order is identified with an arrow.

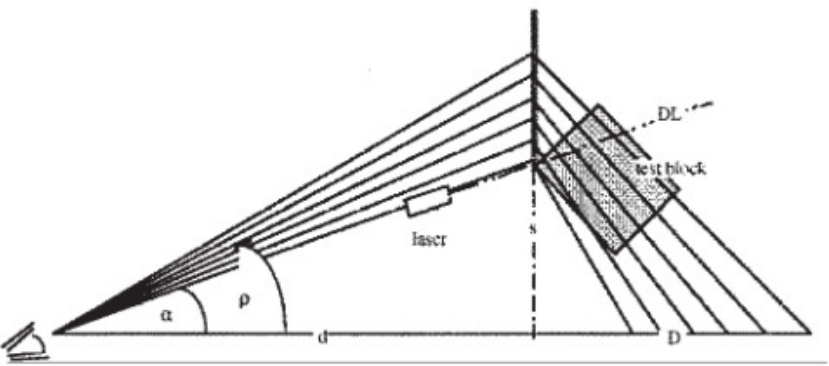

Fig. 9. Setup diagram for experiment with high-frequency grating. Sight lines are ray traced. The target is a step block rotated by $25^{\circ}$ to optimize illumination levels.

The horizontal steps represent a 2.31 increment of range along $D L$. The experiment demonstrates that range sensitivity is equal to or greater than sensitivity to the corresponding lateral dimension.

Occlusion liability can be lowered while range sensitivity is maintained if diffraction range-finder parameters are adjusted properly. For gratings with a pitch shorter than illumination wavelength, consider the change in the incident angle $i$ versus the receiving angle $r$. The function produces a generic relationship, which is graphed in Fig. 12. The point at unity slope, where $i$ equals $r$, is the Bragg angle $\psi$. For values of $r$ above the Bragg angle, a change in $r$ is greater than an equal change in $i$. When proper adjustments are made for input angle and view angle, the grating can serve as a magnifier. ${ }^{9}$ Moreover, if the range finder is designed to use angles centered on the Bragg angle, relatively efficient transmission of light is ensured.

For example, consider the setup illustrated in Fig. 13. We use a grating with a pitch of $400 \mathrm{~nm}$ and a $670-\mathrm{nm}$ laser and set $d=15 \mathrm{~cm}$ and $s=15 \mathrm{~cm}$. The
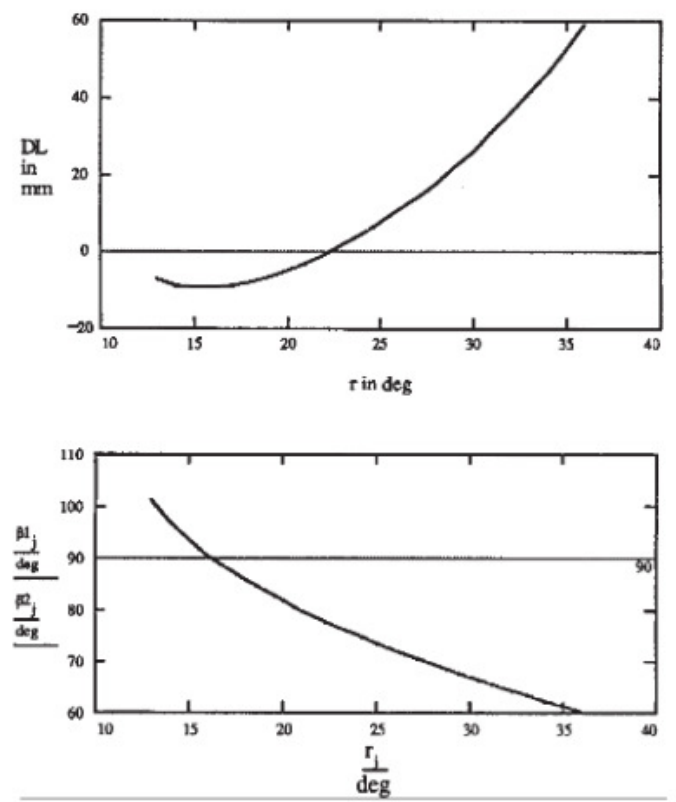

Fig. 10. DL versus receiving angle compared with occlusion liability angle, $\beta$, with a 555-nm grating, where $\alpha=22^{\circ}$ and $s=100$ $\mathrm{mm}$. 

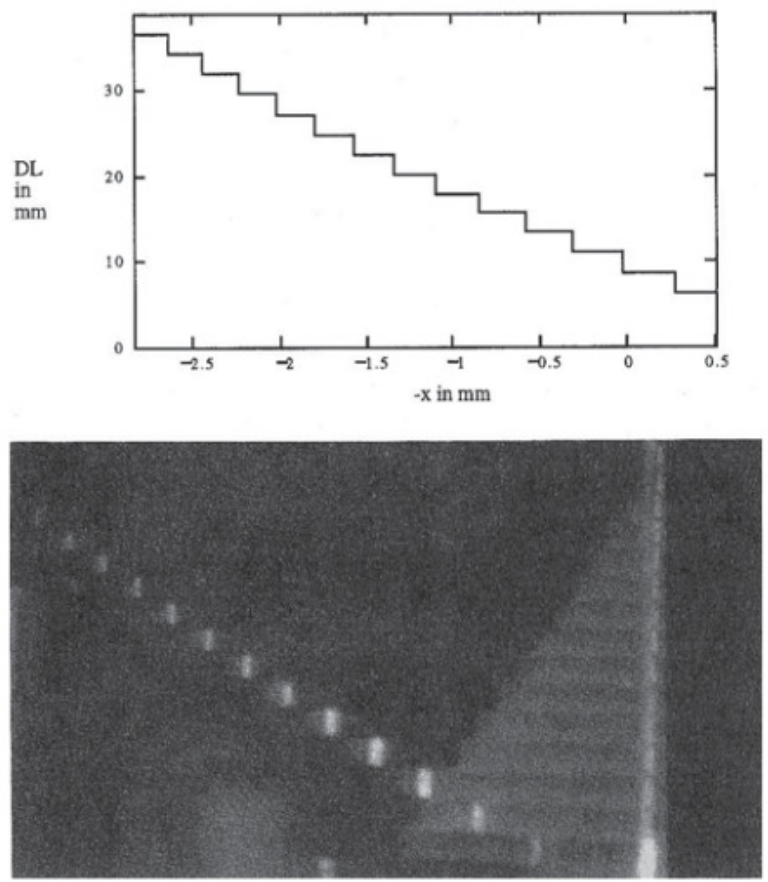

Fig. 11. Comparison of predicted performance with an image that was camera recorded with a high-frequency grating. The camera has a sensor measuring $6.41 \mathrm{~mm}$ in the horizontal, which is given as $\mathrm{x}$ in the graph. The zero-order image appears in the camera image as a vertical line.

laser beam is off axis by $\alpha=48^{\circ}$. As shown in Fig. 14 , this configuration produces an occlusion liability angle of under $20^{\circ}$ throughout the sampled region. Yet range sensitivity is better than that produced by low-frequency gratings with equivalent occlusion immunity. We model the camera image in Fig. 15, assuming a lens of $25 \mathrm{~mm}$ on a 5-mm-wide chip. A camera-recorded image, made with a 6.5 -mm-wide sensor chip, is shown in Fig. 16. It shows artifacts of magnification much like those produced by anamorphic lenses. The extent of magnification can be evaluated by the use of the test block steps. In the near field, the steps appear to be equal in both the horizontal and the vertical directions, that is, in this configuration the diffraction range finder is equally sensitive to lateral and depth dimensions.

The artifacts in Fig. 16 include a relatively short depth of field. Only the near field is in focus. A method of compensation for increasing depth of field is suggested in Section 5 .

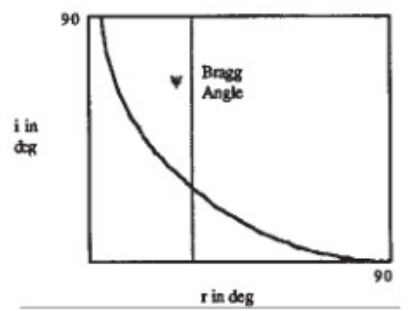

Fig. 12. Generic curve that relates incident and receiving angles for gratings with pitch shorter than incident illumination. At angle $\psi, i=\mathbf{r}$.

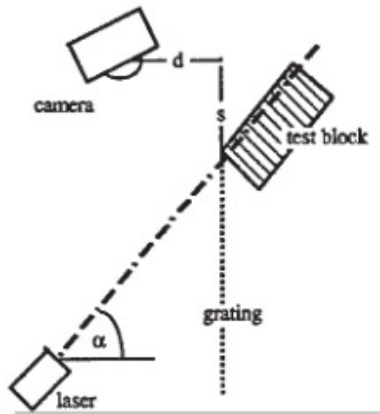

Fig. 13. Configuration for combined occlusion immunity and high range sensitivity

\section{Scheimpflug Condition}

Depth of field plays a role in the diffraction range finder; it must be maximized. The higher-order spectra must be resolved at the detection plane over a wide span of distances from the grating. Given that this depth-of-field problem is the reverse of that of the focus analysis range finder, in which depth of field must be held to a minimum, it is clear that diffraction range finders benefit from the use of wide-angle lenses. Photographers practiced in the art know that wide-angle lenses have the greatest hyperfocal distance, that is, distance from infinity to a foreground point that can be resolved as in focus. The standoff of lens to grating creates a natural fit between all components, requiring no focus adjustment in many implementations of diffraction ranging.

If a long-focal-length lens is used in a diffraction range finder, which could be the case if grating size were to be held to a minimum, the diffraction method lends itself to a special form of focus compensation. When a lens is used to form an image of a sloping object, the object plane, the image plane, and the median plane along the lens will all meet together at a common point. We illustrate this in Fig. 17. This method of focus compensation is called the Scheimpflug condition. ${ }^{10}$

The object plane lies on the line segment $A C$. The image plane lies on the line segment $A D$. The
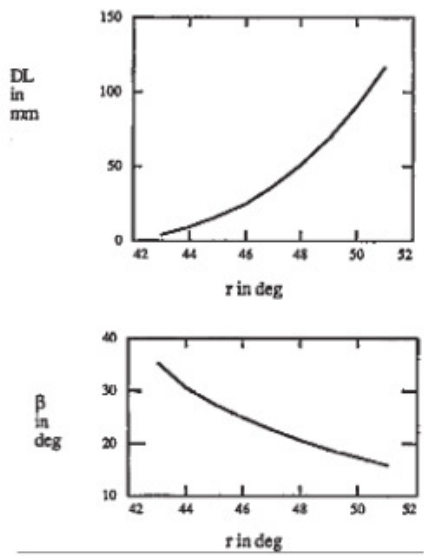

Fig. 14. Receiving angle correlated to range $D L$ and occlusion liability angle $\beta$. 


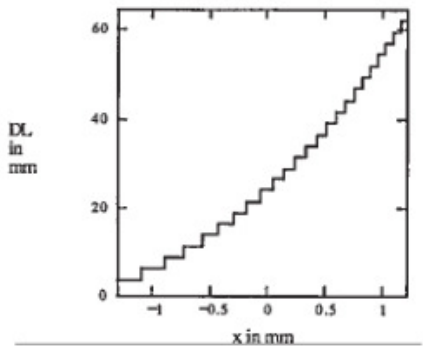

Fig. 15. Camera model prediction assuming a $25-\mathrm{mm}$ lens on a camera $18 \mathrm{~cm}$ from the grating.

median plane passes through the lens and lies on the line segment $A B$. All planes are shown to meet at a common point, $A$, which forms the apex of two right-angle triangles, $A B C$ and $A B D$. These triangles share a common side $(A B)$ whose length is

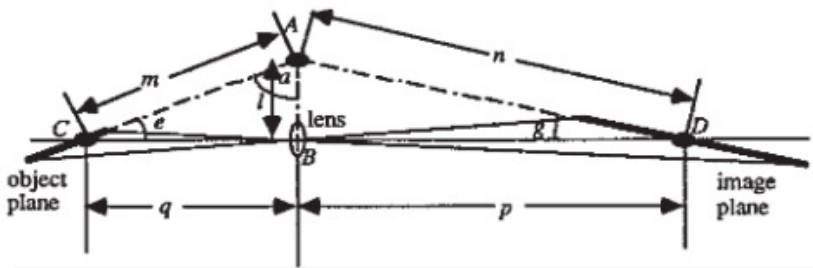

Fig. 17. Geometric model of the Scheimpflug condition.

The position sensor and the target are at arbitrary angles with respect to the diffraction grating. The relation between the angle of the position sensor, $g$, with respect to the main optical axis, $Z D$, the position sensor distance from the lens, object pose (position and orientation), lens focal length, diffraction-grating pitch, diffraction-image order, and illumination wavelength shown in Fig. 18 is

$$
g=\tan ^{-1}\left[\frac{ \pm d\left[n \lambda / p-\sin \left[\tan ^{-1}\left(\frac{t \sin k}{t \cos k+d+\mathrm{q}}\right)\right] \int\left[\cos \left[\tan ^{-1}\left(\frac{t \sin k}{t \cos k+d}\right)\right]\right]^{-2}-1\right\}^{1 / 2}}{\frac{f t \sin k}{t \cos k+d+q}\left(1-\left\{n \lambda / p-\sin \left[\tan ^{-1}\left(\frac{t \sin k}{t \cos k+d+q}\right)\right]^{2}\right]^{1 / 2}\right.}-\frac{t \sin k}{t \cos k+d}\right] .
$$

denoted as $l$. The optical axis of the camera lies on line segment $C D$.

If we let angles $C A B$ be denoted as $a, A C B$ be denoted as $e, B D A$ be denoted as $g$, and assume that we have a simple converging lens of focal length $f$, then

$$
g=\tan ^{-1}\left[ \pm \frac{q}{f}\left(\cos ^{-2} e-1\right)^{1 / 2}-\tan e\right]
$$

must be true. The proof of Eq. (13) appears in Appendix B.

The geometric model of the Scheimpflug condition becomes more interesting when a diffraction grating is inserted between the lens and the object to be imaged (i.e., in front of the camera). A sketch of this model is shown in Fig. 18.

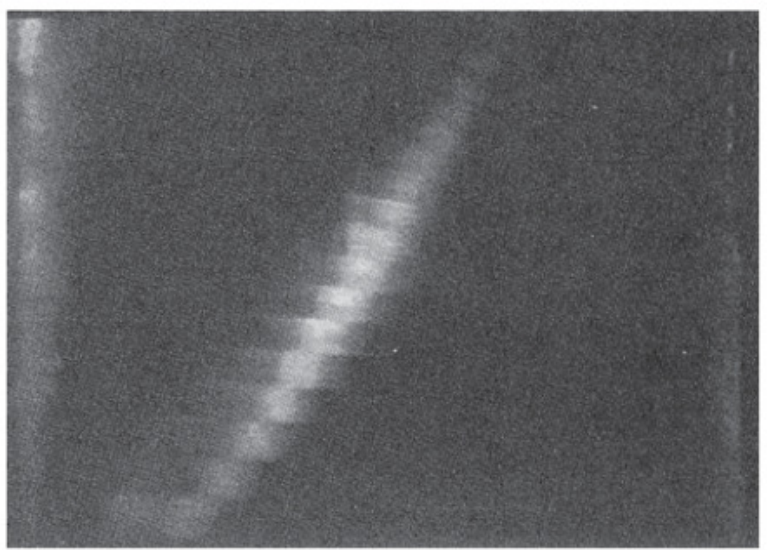

Fig. 16. Camera recorded test block with low occlusion liability and high sensitivity.
The proof of Eq. (14) is given in Appendix B.

\section{Comparison of Diffraction Range Finding with Other Methods}

Multiple-stripe triangulation systems, which use diffraction gratings to project an array of points or lines on a target surface, have been developed. One such system uses multiple-triangulation cameras to resolve ambiguities caused by overlapping lines. ${ }^{11}$ Diffraction range finders, on the other hand, can produce multiple images from a single point, each image having its own perspective view. This simple reversal of function, in which the grating is used to observe a target surface rather than illuminate it, has a variety of advantages over conventional triangulation.

Near-field blindness, which is endemic to all stereoscopic and triangulation devices, can be avoided in

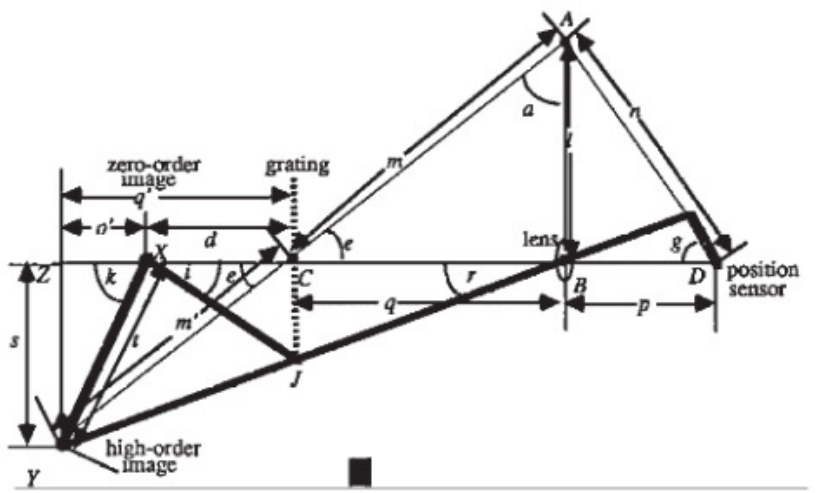

Fig. 18. Geometric model of diffraction and the Scheimpflug condition. 
diffraction range finders, as they can be designed to work to point of contact. Moreover, in diffraction range finders the source of illumination and the receiver can be coaxial. Such a configuration overcomes synchronization problems that affect scanning triangulation sensors. The occlusion problems characteristic of triangulation can be moderated by the use of multiple cameras and relatively narrow baselines between projector and sensor. Our diffraction method can achieve similar benefits with a single camera viewing multiple higher-order images. If the source of illumination is directed off axis, the diffraction method can be combined with triangulation to produce a compound effect that has the features of both methods. Another marriage of technologies could include anamorphic lenses and the diffraction magnification feature we have disclosed here.

Lenses in themselves have the ability to measure range through focus analysis. Like the diffraction method, these methods are monocular, that is, transmitter and receiver can be coaxial. However, compared with the diffraction method, which measures the deflection of a point, the focus-analysis method is computationally expensive. It requires that a measurement be made on a Gaussian spot that can vary widely in brightness, depending on target reflectivity. The computation can be avoided if the spot is mechanically focused to the smallest diameter, but this method is cumbersome for large lenses. Unfortunately, the accuracy of the focus-analysis method is proportional to the size of the lens. The diffraction method is also scaled by the size of the grating, that is, the more distant the point, the larger the grating required for ranging it, but we have demonstrated that plastic embossed holographic gratings can be used. Compared with lenses, even Fresnel lenses, these plastic gratings are very cost effective. Furthermore, no mechanical focusing is required if the diffraction range finder has Scheimpflug compensation.

Interferometric methods of range finding share some of the same underlying physics with our diffraction method, as both rely on the behavior of wave fronts that form constructive peaks and destructive nodes. However, the grating method returns range measurements in absolute coordinates, whereas classical interferometry produces relative measurements that can be ambiguous over discontiguous surfaces. Moreover, although we have used laser light for convenience, the diffraction method does not require coherent illumination. It works perfectly well in incoherent multispectral illumination, provided that a point source can be resolved on the target surface.

Time-of-flight methods of range finding have distinct advantages over diffraction in the far field, but for near-field work, the diffraction method is superior. The diffraction method improves in accuracy inversely with target distance, and there is no cross talk between the transmitter and the receiver as there is with time-of-flight methods.

\section{Conclusion}

Diffraction range finding opens a new application for gratings. The practical need for three-dimensional instrumentation forms a strong motivation for continued investigation of the method. Potential uses exist in microscopy, machine vision, and computer graphics. We have posited a series of geometric relationships that can be used to model diffraction range finders. Work remains to be done in physical optics to model the intensity fields and limits of resolution for gratings used in range-finding applications.

\section{Appendix A}

We have previously shown that

$$
D=d \tan r \frac{\left|1-(n \lambda / p-\sin r)^{2}\right|^{1 / 2}}{n \lambda / p-\sin r} .
$$

Refer to Fig. 3 in the body of the text; the length along a normal from the median extending to the point of the observed higher-order diffraction can be determined by

$$
S=d \tan r .
$$

The side $\Delta s$ to the right triangle formed by $D L$ and $D L_{0}$ can be found by

$$
\Delta s=D L_{0} \tan \alpha .
$$

We use side ratios of similar triangles to express the normal distance from the grating, $D L_{0}$, along the line of illumination projected from a laser:

$$
\frac{D}{S}=\frac{D L_{0}}{S-s+\Delta s} .
$$

Substituting Eqs. (A.2) and (A.3) into Eq. (A.4), we have

$$
\frac{D}{d \tan r}=\frac{D L_{0}}{d \tan r-s+D L_{0} \tan \alpha} .
$$

The distance from the grating along the laser line, $D L$, is

$$
D L=D L_{0} / \cos \alpha .
$$

Solving for $D L_{0}$, we find that

$$
D L_{0}=D L \cos \alpha \text {. }
$$

Substituting Eq. (A.7) into Eq. (A.5), we obtain

$$
\frac{D}{d \tan r}=\frac{D L \cos \alpha}{d \tan r-s+D L \cos \alpha \tan \alpha} .
$$

Solving for $D$, we obtain

$$
D=d \tan r \frac{D L \cos \alpha}{d \tan r-s+D L \cos \alpha \tan \alpha} .
$$

10 May 1995 / Vol. 34, No. 14 / APPLIED OPTICS 
Equating Eqs. (A.1) and (A.9) yields

$$
\begin{aligned}
d \tan r & \frac{\left|1-(n \lambda / p-\sin r)^{2}\right|^{1 / 2}}{n \lambda / p-\sin r} \\
\quad & d \tan r \frac{D L \cos \alpha}{d \tan r-s+D L \cos \alpha \tan \alpha} .
\end{aligned}
$$

Dividing both sides of the equation by $d \tan r$ and simplifying results in

$$
\frac{\left|1-(n \lambda / p-\sin r)^{2}\right|^{1 / 2}}{n \lambda / p-\sin r}=\frac{D L \cos \alpha}{d \tan r-s+D L \sin \alpha} .
$$

Partially solving for DL we can say that
The distance from the camera to the grating along $X$ is given by

$$
d=X \cos r .
$$

Substituting Eq. (A.18) into Eq. (A.15) yields

$$
D L=\kappa \frac{X \cos r \tan r-s}{\cos \alpha-\kappa \sin \alpha} .
$$

We define the angle, $\beta$, as the occlusion liability angle so that

$$
\beta=i-\alpha .
$$

Solving the grating equation (2) for $i$ yields

$$
i=\arcsin (n \lambda / p-\sin r) .
$$

$$
D L=\frac{\left(d \tan r-s+D L \overline{\sin \alpha)\left[1-(n \lambda / p-\sin r)^{2}\right]^{1 / 2}}\right.}{\cos \alpha(n \lambda / p-\sin r)} .
$$

For the sake of notational convenience we use the convention that

$$
\kappa=\frac{\left|1-(n \lambda / p-\sin r)^{2}\right|^{1 / 2}}{n \lambda / p-\sin r} .
$$

Substituting Eq. (A.13) into Eq. (A.12) yields

$$
D L=\frac{(d \tan r-s+D L \sin \alpha) \kappa}{\cos \alpha} .
$$

Solving Eq. (A.14) for $D L$ yields

$$
D L=\frac{\kappa(d \tan r-s)}{\cos \alpha-\kappa \sin \alpha} .
$$

We substitute $\alpha=0$ and $s=0$ into Eq. (A.15) to show that this results in Eq. (A.1):

$$
D L=\kappa d \tan r .
$$

Substituting Eq. (A.13) into Eq. (A.16) results in Eq. (A.1):

$$
D L=d \tan r \frac{\left|1-(n \lambda / p-\sin r)^{2}\right|^{1 / 2}}{n \lambda / p-\sin r}
$$

Substituting Eq. (A.21) into Eq. (A.20) and solving for $\alpha$ yields

$$
\alpha=\arcsin (n \lambda / p-\sin r)-\beta .
$$

Given a camera with focal length $F$, rotated from the normal by angle $\rho$, and with focal plane image displacement $x$, we can use trigonometry to find

$$
r=\rho+\arctan (x / F) .
$$

Substituting Eq. (A.23) into Eq. (A.13) yields

$$
\kappa=\frac{\left(1-|n \lambda / p-\sin | \rho+\left.\arctan (x / F)\right|^{2}\right)^{1 / 2}}{n \lambda / p-\sin [\rho+\arctan (\mathrm{x} / \mathrm{F})]}
$$

Substituting Eq. (A.23) into Eq. (A.15) yields

$$
D L=\frac{\mid d \tan (\rho+\arctan (x / F) \mid-s) k \cos \alpha}{1-k \tan \alpha} .
$$

Substituting Eq. (A.24) into Eq. (A.25) yields

$$
D L=\frac{\{d \tan [\rho+\arctan (x / F)]-s\}\left[\frac{\left(1-\left\{n \lambda / p-\sin \left[\rho+\left.\arctan (x / F)\right|^{2}\right)^{1 / 2}\right.\right.}{n \lambda / p-\sin [\rho+\arctan (x / F) \mid}\right]}{\cos \alpha-\left[\frac{\left(1-\left\{n \lambda / p-\sin \left[\rho+\left.\arctan (x / F)\right|^{2}\right)^{1 / 2}\right.\right.}{n \lambda / p-\sin [\rho+\arctan (x / F) \mid}\right] \sin \alpha} .
$$

Simplifying yields

$$
D L=\frac{\left\{d \operatorname { t a n } [ \rho + \operatorname { a r c t a n } ( x / F ) | - s \} \left(1-\left\{n \lambda / p-\sin \left[\rho+\left.\arctan (x / F)\right|^{2}\right)^{1 / 2}\right.\right.\right.}{n \lambda / p-\sin |\rho+\arctan (x / F)|] \cos \alpha-\sin \alpha} .
$$


Appendix B: Proof of the Scheimpflug Condition

In this section we prove that the relation for the film-plane angle for the Scheimpflug condition as a function of the object pose and the lens focal length is

$$
g=\tan ^{-1}\left[ \pm \frac{q}{f}\left(\cos ^{-2} e-1\right)^{1 / 2}-\tan e\right]
$$

where $f$ is the focal length of the lens, $e$ is the angle the target makes with respect to the optical axis, $q$ is the distance from the lens to the target, and $g$ is the angle the sensor makes with respect to the optical axis. In the subsequent subsection we combine this result with diffraction.

A geometric model of the Scheimpflug geometry is shown in Fig. 17.

B.1. Proof

For right-angle triangle $A B C$

$$
\begin{aligned}
l^{2} & =m^{2}-q^{2}, \\
1 / q & =\frac{\tan e}{l}, \\
m & =q / \cos e .
\end{aligned}
$$

For right-angle triangle $A B D$,

$$
\begin{aligned}
l^{2} & =n^{2}-p^{2}, \\
1 / p & =\frac{\tan g}{l}, \\
n & =p / \cos g .
\end{aligned}
$$

For a simple converging thin lens of focal length $f$, we invoke the Gaussian lens formula:

$$
1 / f=1 / p+1 / q
$$

Substituting Eqs. (B.2) and (B.4) into Eq. (B.5) and solving for $g$ yields

$$
g=\tan ^{-1}(1 / f-\tan e) .
$$

Equating Eq. (B.1) with Eq. (B.3) results in

$$
l^{2}=n^{2}-p^{2}=m^{2}-q^{2} .
$$

Substituting Eqs. (B.4a) and (B.2a) into Eq. (B.6a) yields

$$
l^{2}=(p / \cos g)^{2}-p^{2}=(q / \cos e)^{2}-q^{2}
$$

Simplifying Eq. (B.7) yields

$$
l= \pm p\left(\cos ^{-2} g-1\right)^{1 / 2}= \pm q\left(\cos ^{-2} e-1\right)^{1 / 2} .
$$

We substitute Eq. (B.7a) into Eq. (B.6) to obtain

$$
g=\tan ^{-1}\left[ \pm \frac{q}{f}\left(\cos ^{-2} e-1\right)^{1 / 2}-\tan e\right]
$$

Equation (B.8) shows the film-plane angle for the Scheimpflug condition as a function of the object pose and the lens focal length.

Q.E.D.

\section{B.2. Diffraction and the Scheimpflug Condition}

In this section we introduce a diffraction grating into the camera. Using the diffraction image from the position sensor, we are able to compute the range of the object.

The purpose of this section is to compute at what angle to place the position sensor inside the camera, given the position sensor distance from the lens, object pose (position and orientation), lens focal length, diffraction-grating pitch, diffraction-image order, and illumination wavelength. The construction is shown in Fig. 18.

The diffraction grating lies on the line segment $J C$. A point source of light at location $X$ is seen by the position sensor as having a high-order diffraction image that appears to be located at point $Y$. In fact, the point source of light at location $X$ emits a ray that lies on line segment $X J$ and is bent by the diffraction grating to lie on line segment $J B$, passing through the lens. The length of the object $t$ is known. Also known is its angle $k$ with respect to the optical axis. In the following proof, we make use of these parameters and of the fact that right-angle triangle $X Y Z$ has a side in common with right-angle triangle $B Y Z$.

Triangle $X J B$ is subject to the diffraction equation:

$$
\sin i+\sin r=n \lambda / p
$$

where $n$ is the diffraction order, $\lambda$ is the wavelength, and $p$ is the grating pitch. The following equation is identical to Eq. (3) of Section 3 with the exception that there are some notational substitutions. These differences are needed to account for the new Scheimpflug construction shown in Figure 18:

$$
d=\frac{\left.q \tan r \mid 1-(n \lambda / p-\sin r)^{2}\right]^{1 / 2}}{n \lambda / p-\sin r} .
$$

Solving Eq. (B.10) for $q$ results in

$$
q=\frac{d(n \lambda / p-\sin r)}{\tan r\left|1-(n \lambda / p-\sin r)^{2}\right|^{1 / 2}} .
$$

In addition, because the Scheimpflug condition still applies, Eq. (B.8) must still be true, that is,

$$
g=\tan ^{-1}\left[ \pm \frac{q}{f}\left(\frac{1}{\cos ^{2} e}-1\right)^{1 / 2}-\tan e\right]
$$


Substituting Eq. (B.11) into Eq. (B.8) yields

$$
g=\tan ^{-1}\left\{\frac{ \pm d(\gamma-\sin r)\left(\cos ^{-2} e-1\right)^{1 / 2}}{f \tan r\left|1-(\gamma-\sin r)^{2}\right|^{1 / 2}}-\tan e\right],
$$

where $\gamma=n \lambda / p$ for notational convenience. As triangle $X Y Z$ is a right-angle triangle and as we know the angle of the object, $k$, and its length, $t$, we can show that

$$
\begin{gathered}
s=t \sin k, \\
o^{\prime}=t \cos k .
\end{gathered}
$$

By basic trigonometry we can see that

$$
s=q^{\prime} \tan e
$$

must also be true. Equating Eqs. (B.13) and (B.14), we may solve for $e$ :

$$
e=\tan ^{-1}\left(\frac{t \sin k}{q^{\prime}}\right)
$$

It can be seen from Fig. 18 that

$$
q^{\prime}=o^{\prime}+d \text {. }
$$

Substituting Eqs. (B.14) and (B.17) into Eq. (B.16) yields

$$
e=\tan ^{-1}\left(\frac{t \sin k}{t \cos k+d}\right) .
$$

Finally, we observe that right-angle triangle $B Y Z$ has a side in common with right-angle triangle $\mathrm{XYZ}$ so that

$$
r=\tan ^{-1}\left(\frac{s}{q^{\prime}+q}\right) .
$$

Substituting Eqs. (B.15), (B.16), and (B.17) into Eq. (B.19) results in

$$
r=\tan ^{-1}\left(\frac{t \sin k}{t \cos k+d+q}\right) .
$$

Substituting Eqs. (B.18) and (B.20) into Eq. (B.12) results in an equation that yields the angle of the position sensor as a function of the distance of the object from the grating, $d$, the distance of the grating from the lens, $q$, the diffraction grating pitch, illumination wavelength, diffraction order, focal length, object length and object angle:
The authors thank Don Winrich for assistance in some of the derivations found in this article. This research was performed, in part, on grants from the National Science Foundation and the New York State Science and Technology Foundation.

\section{References and Notes}

1. L. H. Bieman, "Designer's handbook-three-dimensional machine vision," Photon. Spectra 22(5), 81-92 (1988); D. Braggins, "3-D inspection and measurement: solid choices for industrial vision," Adv. Imaging 9(10), 36-39 (1994); R. L. Cromwell, "Sensors and processors enable robots to see and understand," Laser Focus World 29(3), 67-82 (1993); K. G. Harding, "Current state-of-the-art of contouring techniques in manufacturing," in Proceedings of ICALEO 1989, Proc. Soc. Photo-Opt. Instrum. Eng. 70, 7-11 (1989); Y. Shirai, Three-Dimensional Computer Vision (Springer-Verlag, New York, 1987); T. C. Strand, "Optical three-dimensional sensing for machine vision," Opt. Eng. 24, 33-40 (1985); T. T. Wohlers, "3D digitizers," Comput. Graphics World X(7), 73 (1992); T. T. Wohlers, "3D digitizing systems," Comput. Graphics World 17(24), 59-61 (1994); H. D. Wolpert, "Autoranging/autofocus a survey of systems," Photon. Spectra 21(6), 165, 21(8), 127, 21(9), 133-142 (1987).

2. P. Wieland and H. J. Tiziani, "Computer synchronized 3-D triangulation sensor for robot vision," in Optics, Illumination, and Image Sensing for Machine Vision VII, D. J. Svetkoff, ed., Proc. Soc. Photo-Opt. Instrum. Eng. 1822, 168-174 (1992); M. Rioux, "Laser range finder based on synchronized scanners," Appl. Opt. 23, 3837 3844 (1984).

3. F. A. Jenkins and H. E. White, Fundamentals of Optics (McGraw-Hill, New York, 1950), p. 327.

4. T. DeWitt, "Novel methods for the acquisition and display of three-dimensional surfaces," in Optical and Digital Pattern Recognition, H. Liu and P. S. Schenker, eds., Proc. Soc. Photo-Opt. Instrum. Eng. 754, 55-63 (1987).

5. Equation (3) was first published in Ref. 4. Equation (6) was first published by T. DeWitt in "A guide to 3-D surface Acquisition," in Proceedings of SCAN 90 (Symposium of the Computers in the Arts Network, University of the Arts, Philadelphia, Penn., 1990), pp. 40- 46.

6. T. DeWitt, "Range finding by diffraction," U.S. patent 4,678,324 (7 July 1987); European patent 0,343,158 B1 (25 May 1994).

7. J. Woo, principal investigator, "Near field ranging for robotic vision applications," NSF SBIR Final Rep., Grant ISI-8761135 (Interscience, New York, 1989).

8. H. Stark, "The field produced by a grating in the Fresnel zone of a point source," commissioned report to Raytel, Inc., Troy, N.Y., 1985. (This document was the basis of Ref. 7. Stark's consultance was solicited by T. DeWitt, who was then at Raytel.)

9. J. Parker, "Sketch for a possible probe design," Box 83,

$$
g=\tan ^{-1}\left[\frac{\left. \pm d\left[n \lambda / p-\sin \left[\tan ^{-1}\left(\frac{t \sin k}{t \cos k+d+q}\right)\right]\right\}\left[\cos \left[\tan ^{-1}\left(\frac{t \sin k}{t \cos k+d}\right)\right]\right]^{-2}-1\right\}^{1 / 2}}{\frac{f t \sin k}{t \cos k+d+q}\left(1-\left\{n \lambda / p-\sin \left[\tan ^{-1}\left(\frac{t \sin k}{t \cos k+d+q}\right)\right]\right]^{2}\right]^{1 / 2}}-\frac{t \sin k}{t \cos k+d}\right] .
$$

Q.E.D. 
Ancramdale, New York 12503 (private communication, 1 December 1993).

10. R. Kingslake, Optics in Photography, (Society for PhotoOptical Instrument Engineers, Bellingham, Wash., 1992).
11. S. J. Gordon, "Real-time 3D range sensor," in Industrial Applications of Optical Inspection, Metrology, and Sensing, G. M. Brown, K. G. Harding, and H. P. Stahl, eds., Proc. Soc. Photo-Opt. Instrum. Eng. 1821, 312 (1992). 